\title{
La décentralisation des politiques publiques en matière d'emploi au Québec : vers de nouvelles perspectives de recherche
}

\author{
Jean-François Simard ${ }^{1}$ \\ Université Laval
}

Après un demi-siècle de diffusion massive des sciences sociales au Québec, tout particulièrement dans les vastes champs des relations industrielles et de la sociologie qui ont abondamment traité du travail à travers sa quadruple fonction de production, de rémunération salariale, d'intégration sociale et d'innovation technologique; plus de quatre décennies après la tenue du Bureau d'aménagement de l'Est du Québec (BAEQ) qui a vraiment lancé la réflexion sur la décentralisation des politiques publiques; et après 40 ans de sciences régionales et de sociologie du développement qui ont singularisé la région dans sa spécificité sociodémographique et ses besoins socio-économiques, force nous est de constater que la question de la décentralisation des politiques publiques en matière d'emploi demeure un chantier scientifique relativement vierge au Québec. Cela est d'autant plus surprenant que la dernière décennie a vu naître une floraison d'organisations régionales et locales œuvrant à cette tâche.

Cet article propose une réflexion épistémologique sur la question de l'emploi. Il en appelle à une refondation de la décentralisation des politiques publiques de l'emploi comme objet de recherche davantage enraciné dans l'historicité québécoise. Pour ce faire, trois grands défis nous incombent : assumer la dialectique de l'objet - car on qualifie en effet de dialectique toute pensée qui se montre sensible à ses propres contradictions - comprendre le déficit structurel de l'emploi qui perdure entre nos régions et expliquer les particularités émergentes du modèle québécois.

\section{Premier défi : assumer la dialectique de l'objet}

La décentralisation des politiques publiques comme sujet d'étude recèle une dualité idéologique et une ambiguïté terminologique dont le politique et le chercheur doivent maîtriser les paramètres. À prime abord, vouloir décentraliser une politique publique, c'est vouloir décentraliser la res publica. Autrement dit, c'est avoir l'intention de subtiliser quelque chose au public, une chose qui, par définition, appartient à la collectivité nationale pour le remettre entre les mains d'une collectivité régionale. Il s'agit en soi d'un projet révolutionnaire, parce qu'il remet en cause un ordre politique établi au fil des siècles. De fait, l'idée de la décentralisation va à contresens historique de l'idée de l'État-nation parce que cette dernière s'est lentement construite aux dépens des pouvoirs régionaux et des villes-républiques.

Dans ce champ de recherche s'affronte, ou du moins cohabite en quête d'un équilibre souvent précaire, la logique de la région par rapport à celle de l'Étatnation. Le trait d'union entre les deux pôles de ce même continuum s'incarne à travers la notion clé de notre cadre conceptuel : la décentralisation. Politologues, sociologues, administrateurs publics et juristes ont défini la décentralisation jusqu'à plus soif. L'objectif de cet article n'est pas d'en faire une recension exhaustive, mais plutôt d'attirer l'attention sur la fonction subversive de l'idéologie de la décentralisation. Car, en effet, abordé dans une perspective de gouvernance, la question de la décentralisation, qui d'ailleurs s'appuie sur le paradigme de la subsidiarité, 
postule une meilleure cohésion entre l'éthique politique et la gestion administrative lorsque le pouvoir de décision est situé à proximité du lieu d'action. Ainsi, la décentralisation a comme base constitutive de son discours une opposition systémique à l'État dans sa dimension totalitaire.

La région - elle aussi concept extensible et riche de sens, qui a donné lieu à une kyrielle de définitions est ici vue comme un espace de médiation idéal entre l'acteur et le planificateur gestionnaire. Les dimensions du dialogue (mobilisation des acteurs individuels et institutionnels qui composent la société civile), d'adaptation (respect de l'identité locale) et de flexibilité (rejet des politiques mur à mur) sont également inhérentes au concept de décentralisation. Mentionnons enfin que cette dernière est aussi associée à une démocratie participative, partenariale et parfois corporative largement étayée dans la littérature. La décentralisation repose sur un apprentissage de la manière de vivre ensemble et un processus de socialisation politique qui valorise les approches horizontales du territoire, plutôt que les approches sectorielles et la logique des filières de l'État ${ }^{2}$.

Ainsi posée, la logique de la décentralisation se heurte à celle de l'État-nation. D'abord, parce que cette dernière renvoie à la culture démocratique de la représentation dont l'un des grands fondements est l'imputabilité, lui-même originaire du concept anglais d'accountability. Déjà présente dans la Magna Carta et largement à l'origine de la révolution américaine, la question est universellement la même: devant qui répond-t-on de la gestion des deniers publics? Penser l'opérationnalisation de notre objet réfère inévitablement à une question de légitimité. On ne peut conceptualiser la décentralisation en faisant l'économie d'une réflexion sur les modes de régulation politique d'une gouvernance locale intégrant les fonctions d'imputabilité.

La dynamique de la décentralisation postule aussi le dépassement traditionnel de la négation du local dans le modèle de développement économique keynésien instauré chez nous surtout depuis la Révolution tranquille. Un présupposé normatif, plutôt négatif, a longtemps affecté la région comme espace de vie économique. Dans cette perspective, le local se pré- sente comme une autre notion clé de notre cadre conceptuel. Dans la foulée de la Deuxième Guerre mondiale, la conception territoriale référait presque exclusivement à l'espace national. Le local était associé, dans l'imaginaire collectif des décideurs publics, à un monde dépassé, en retard sur la modernité administrative et source de résistance aux changements sociaux ${ }^{3}$.

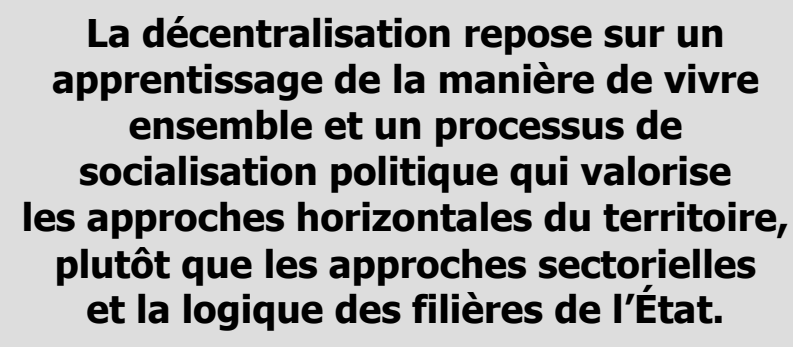

La montée du discours sur la décentralisation repose sur le double postulat que le développement local devient plus important dans le nouvel environnement économique que dans l'ancien et que le local dont il est maintenant question s'est renouvelé du point de vue de l'originalité et de la vitalité des liens sociaux qui le structurent ${ }^{4}$. À cet égard, mentionnons également que, dans sa dimension opératoire, la décentralisation des politiques de l'emploi se caractérise souvent par une valorisation de l'a priori social de l'activité économique - économie sociale. L'économiste Hirschman ${ }^{5}$ a démontré clairement que la logique du développement échappe souvent à l'analyse économique conventionnelle en soulignant l'importance des effets de liaisons, ces mécanismes sociaux générateurs et mobilisateurs d'énergie, de capital et d'esprit d'entreprise pour expliquer la croissance. En bref, l'idéologie de la décentralisation répond à une révolution conceptuelle du local comme lieu de planification et de régulation de l'action socioéconomique.

La décentralisation suppose aussi que l'espace national soit composé de sous-ensembles d'appartenance identitaires et territoriaux définis en fonction d'un certain nombre d'indicateurs que la géographie sociale a contribué à développer (géomorphologie, densité de la population, histoire locale, etc.). Elle s'inscrit dans un processus politique, animé par une vision pluraliste de la société par lequel les groupes 
d'intérêt de différentes natures négocient, dans un rapport de force, l'équilibre du pouvoir pour en : « arriver à l'idéal politique aristotélicien : un ordre négocié qui crée l'unité à partir de la diversité »" ${ }^{6}$. Alors que 1'État nation se pense comme un phénomène social total, comme un tout culturellement homogène, irréductible à la simple addition de ses constituantes, dont l'intégrité territoriale et l'autorité ne sont pas négociables. Le célèbre manifeste de l'abbé Sieyès (1748-1836) résume bien cette conception: "La nation existe avant tout, elle est à l'origine de tout. Sa volonté est toujours légale, elle est la loi elle-même ${ }^{7}{ }^{7}$. Penser la décentralisation, c'est aussi se demander jusqu'où pousser l'autonomie politique et fiscale d'une région sans pour autant briser l'esprit national. À n'en pas douter, la question de la décentralisation est au cœur de l'actuelle mutation de la conception de l'État-nation.

\section{L'idéologie de la décentralisation répond à une révolution conceptuelle du local comme lieu de planification et de régulation de l'action socio-économique.}

Les politiques de l'emploi et la question du travail ne sont pas à l'épreuve de ces considérations de philosophie politique. À titre d'exemple, nous savons que le seuil de pauvreté varie substantiellement d'une région à l'autre du Québec. Dans ce contexte, des leaders régionaux pourraient-ils réclamer, au nom de la reconnaissance de la spécificité de leur territoire, une variation interrégionale du salaire minimum, sans pour autant nier à l'État son rôle d'assumer l'équité et la justice entre les régions et les travailleurs de ces mêmes régions ? Serait-ce là un recul ou un progrès social ? Derrière la question de la décentralisation s'en cache une autre, celle de la citoyenneté, qui exige théoriquement l'égalité devant la loi (isocratia).

La décentralisation des politiques de l'emploi soulève de nombreuses questions d'éthique politique. Jusqu'où peut-on pousser l'autonomie d'une région eu égard à ses besoins particuliers en matière de formation, de perfectionnement et de requalification de sa main-d'œuvre, dans un même espace d'éducation nationale? Jusqu'où peut-on transférer la responsabilité d'élaborer et de mettre en œuvre les politiques en matière d'emploi, de soutien à l'entreprenariat local, à l'immigrant investisseur, au développement des parcs industriels, sans dislocation de la logique nationale du marché du travail ? Les interactions nombreuses et complexes entre le développement local et des problèmes tels que la réinsertion professionnelle, l'employabilité des travailleurs peu qualifiés, la reconversion des vieilles unités industrielles - souvent localisées sur des sols qu'elles ont lourdement contaminés - et la lutte à la pauvreté dans les quartiers défavorisés font de la décentralisation des politiques publiques de l'emploi un enjeu scientifique d'une très grande pertinence sociale. La qualité de l'emploi influence directement la qualité du capital social que l'on peut observer dans une communauté. La relation symbiotique entre le travail et certaines municipalités monoindustrielles est l'exemple le plus radical de cette coexistence. La difficulté de Murdochville de survivre à la fermeture de Noranda en offre une contemporaine et triste illustration.

L'approfondissement de nos connaissances au chapitre de cette relation toute particulière qui s'instaure entre la gouvernance locale et la question de l'emploi doit se concevoir non seulement dans le continuum centralisation/décentralisation, mais aussi se penser dans le continuum interventionnisme/libre arbitre du marché. À cet égard, cette question de l'emploi souffre encore de l'absence d'analyses socio-économétriques rigoureuses qui pourraient nous éclairer sur les retombées effectives de l'intervention de l'État et des collectivités régionales (Political hand) sur le marché du travail ${ }^{8}$. L'élaboration d'indicateurs locaux quant aux politiques de l'emploi constitue un autre axe de recherche qui attend les spécialistes.

\section{Deuxième défi : expliquer le déficit structurel interrégional de l'emploi}

Un facteur d'ordre structurel devrait inciter les spécialistes des relations industrielles, de la sociologie du travail et des sciences régionales à s'intéresser, encore plus qu'ils ne l'ont fait maintenant, à la question de la décentralisation des politiques publiques en matière d'emploi. Il s'agit de la disparité 
interrégionale du taux de chômage qui perdure entre plusieurs régions du Québec et la moyenne nationale, malgré une décennie relativement bonne au niveau de la création d'emplois.

Après plus de dix ans de croissance du Produit intérieur brut (PIB) québécois et une résorption globale du taux de chômage, qui est passé de $14 \%$ à $8 \%$, entre 1993 et 2002, plusieurs régions du Québec affichent encore un taux de chômage supérieur à $10 \%$. Cela est notamment le cas de la Mauricie (11,6\%), de l'Abitibi-Témiscamingue $(10,1 \%)$, de la Côte-Nord (12,3\%), de la GaspésieIle-de-la-Madelaine $(15,8 \%)$ et du Centre du Québec (10,1\%); alors qu'une région comme celle de Chaudière-Appalache affiche un taux de chômage de $6,1 \%$ (compilation de l'Institut de la statistique du Québec, février 2002).

Comment expliquer ce phénomène au moment même où le Québec, dans son ensemble, a atteint un sommet historique quant à son taux d'emploi en 2002 en dépassant la barre des $60 \%$ ? Après une décennie d'expansion économique, notamment caractérisée par une montée significative de notre production destinée à l'exportation, la main invisible d'Adam Smith, le principe du trickle down américain, la théorie des pôles satellitaires de développement d'Higgins, Martin, Reynauld et les bienfaits du Welfare State auraient pourtant eu amplement le temps de faire sentir leurs effets jusque dans la périphérie des régions dites périphériques. À cette question, certains esprits empressés pourraient répondre que, lorsqu'elles auront rejoint une économie de deuxième et troisième transformation, les régions à fort taux de chômage retrouveront la voix de la prospérité. Mais ces derniers ne feraient que déplacer le problème. Pourquoi ces régions n'y sont-elles pas encore parvenues? Y aurait-il une culture de la différence qui explique la difficulté de leur rattrapage économique ? Quoi qu'il en soit, un nombre grandissant d'auteurs plaide pour «un enrichissement de nos connaissances et pour le renouvellement du cadre général de la géo-économie québécoise ${ }^{9}$. La double approche du libre marché et de l'Étatprovidence a subi l'épreuve du temps. Plusieurs acteurs locaux sont ainsi tentés de résorber le déficit structurel de l'emploi de leur collectivité par le déploiement d'une stratégie locale caractérisée par une décentralisation asymétrique des politiques de l'emploi.

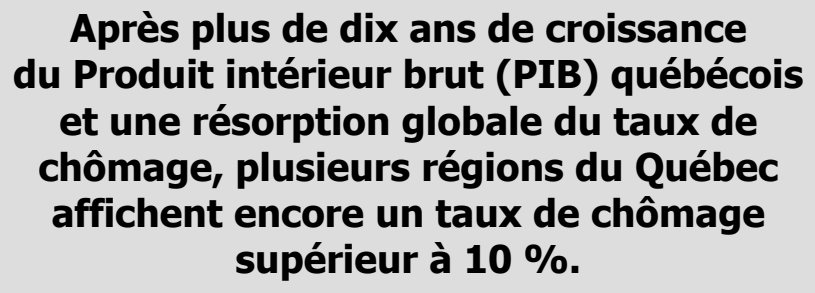

Malgré un taux de chômage qui avoisine le seuil psychologique des $10 \%$, nous nous berçons encore dans la douce illusion de la fin du chômage à court terme. Emploi-Québec prévoit d'ailleurs que plus de 600000 emplois seront disponibles d'ici 2006. Cependant, rien ne permet d'espérer une réduction de l'écart structurel interrégional du taux de chômage. À moyen terme, les stratégies endogènes de création d'emplois resteront d'une incontournable actualité.

\section{Troisième défi : comprendre la spécificité du modèle québécois}

L'essentiel de la littérature internationale qui traite de la décentralisation des politiques de l'emploi aborde généralement cet objet de recherche sous l'angle d'un transfert de juridiction et de responsabilités administratives de l'État fédéral central vers ses entités fédérées ${ }^{10}$. Cette littérature a largement bénéficié des travaux de l'Organisation de coopération et de développement économiques (OCDE) à travers le programme LEED (Local Economic Employment Development $)^{11}$. Mais ces travaux, quoique d'une richesse exceptionnelle, ne tiennent pas compte de la réalité politique québécoise qui, bien que d'appellation nationale, n'a que les attributs constitutionnels d'une législature provinciale. Malgré son statut, le Québec a poussé plus loin que la plupart des pays de l'OCDE ses tentatives de décentralisation et de régionalisation de l'emploi. Au cours des dernières années ont émergé un certain nombre d'organisations de services dans une perspective de partenariats entre l'État et les communautés locales (Enabling State et empowerment local) pour relever le défi de la création d'emplois. Mentionnons notamment les 
Carrefours jeunesse-emploi (CJE), les centres locaux de développement (CLD), les centres locaux d'emploi (CLE), les corporations de développement économique communautaire (CDEC), les sociétés locales d'investissement dans le développement de l'emploi (SOLIDE) et les comités des partenaires du marché du travail.

Ces institutions participent à différents processus de régulation politique qui ne sont pas à proprement parler de la décentralisation. Certaines organisations comme les CLE relèvent davantage d'un mouvement de déconcentration - ce système dans lequel le pouvoir de décision est exercé par des agents opérant sur place mais soumis à l'autorité centrale. Cette perspective se confirme dans la Convention de performance et d'imputabilité d'Emploi-Québec (2002, p. 19) dans laquelle on peut lire qu'« Emploi-Québec élabore et propose ses cibles de résultats à partir des orientations gouvernementales et ministérielles ». Comme leur nom l'indique, les comités des partenaires du marché du travail relèvent pour leur part d'une démarche de concertation et de partenariat, tandis que les SOLIDE se caractérisent davantage par une entente administrative. Les CJE, les CDEC et les CLD s'inscrivent dans un processus de décentralisation administrative. Ceux-ci sont dirigés par des conseils d'administrations élus au cours d'assemblées générales annuelles par des collèges électoraux représentant divers secteurs de la société civile (mouvements coopératifs, patronaux, ouvriers, agricoles, etc.) Contrairement aux CLE, le financement de ces organisations n'est pas exclusivement tributaire de l'État.

\section{Au cours des dernières années ont émergé un certain nombre d'organisations de services dans une perspective de partenariats entre l'État et les communautés locales pour relever le défi de la création d'emplois.}

De nombreuses questions se posent sur l'efficience de telles organisations de services. Sont-elles trop nombreuses ? Y a-t-il complémentarité ou duplicité dans l'action ? Un modèle québécois est-il en train de naître ? Ces organisations auraient-elles besoin d'une marge de manœuvre encore plus grande de la part de l'État? Quels sont les nouveaux transferts de compétences réclamés ? Quelle est la contribution effective de ces institutions au regard du développement économique local, et tout particulièrement de la création de l'emplois? Qu'y ajouterait la décentralisation? Quels sont les indicateurs socioéconomiques qui permettraient une telle évaluation? Quelle est la participation de la société civile dans l'élaboration des stratégies locales de création d'emplois ? Y décèle-t-on l'émergence d'une nouvelle forme de gouvernance locale ? Comment les chambres de commerce et le patronat local cohabitent-ils avec cette apparente démocratisation du développement économique local ? Comment les grands mouvements syndicaux réussissent-ils à dépasser le strict intérêt corporatif de leurs membres et à représenter l'ensemble du mouvement ouvrier? En quoi la décentralisation des politiques publiques de l'emploi est-elle plus sensible au vieillissement de la population et au déclin démographique - pour ne nommer que ces facteurs - qu'une approche dite centralisée?

Malgré la contribution de certains auteurs ${ }^{12}$, la littérature scientifique fournit encore peu de réponses circonstanciées à ces interrogations. Bien que dans leur quête constante et compréhensible de légitimité, des acteurs politiques aient pu faire l'économie d'une telle rationalisation, d'un point de vue scientifique il est difficile de tirer quelle que conclusion que ce soit quant à l'impact objectivement repérable de telles structures, faute de démonstrations probantes. Voilà autant de chantiers d'investigation qui se présentent aux chercheurs.

\section{Conclusion}

Le discours scientifique dominant de la fin des années 1990 sur la question de l'emploi a pu avoir un effet démobilisant auprès de la recherche scientifique. Comme si la question de l'emploi était réglée pour de bon. Alors que Rifkin ${ }^{13}$ prophétisait la fin du travail, que Boyer et Durand ${ }^{14}$ postulaient la fin $\mathrm{du}$ compromis social fordiste, et que Castel ${ }^{15}$ soutenait la fin du salariat comme principal mode de régulation socio-économique, la question de la création de l'emploi aura pu, un certain temps, perdre tout attrait comme objet de recherche. Comme y 
faisait référence Angers ${ }^{16}$, ces discours avaient en commun de proposer une forme de malthusianisme $\mathrm{du}$ salariat. Conséquemment, ces approches ont débouché sur des réflexions portant sur la répartition des heures de travail disponible plutôt que sur la création proprement dit de nouveaux emplois ${ }^{17}$. Néanmoins, le travail rémunéré demeure un fondement de la cohésion communautaire pour laquelle les sciences sociales seront longtemps interpellées. Dans leur grille d'analyse, celles-ci ne peuvent rester captives de modèles de pensée importés d'ailleurs.

\section{La décentralisation des politiques de l'emploi doit donc se penser à l'aune de nos récentes expériences collectives.}

Jacques Grand'Maison ${ }^{18}$ nous rappelle qu' « on ne peut faire du neuf durable si on efface les traces des chemins parcourus ». Dans sa tentative de reingenering de l'État, le nouveau gouvernement du Québec ne doit pas immédiatement succomber à la tentation du tabula rasa, si fréquente dans nos choix sociaux. Il y a ici convergence entre l'approfondissement de la connaissance scientifique et l'ethos politique. La décentralisation des politiques de l'emploi doit donc se penser à l'aune de nos récentes expériences collectives, dans une mise à distance de notre héritage social et par le biais d'une évaluation critique de nos actions et de leurs retombées.

\section{Notes et références}

1 L'auteur est Ph.D. en sociologie. Il a été ministre du gouvernement Landry. Il est présentement chargé de cours en relations industrielles à l'Université Laval.

2 Teisserenc, P. (1994). Les politiques de développement local, approche sociologique, Paris, Économica, coll. Collectivités territoriales.

3 Dumont, F. (1966). Le pouvoir dans la société canadiennefrançaise, Québec, Les Presses de l’Université Laval.

4 Lévesque, B. (1999). « Développement local et économie sociale : éléments incontournables du nouvel environnement », Économie et Solidarités, vol. 30, nº 1, p.111-126.
5 Hirschman A. (1958). The Strategy of Economic Development, New Haven, Yale University Press.

6 Morgan, G. (1989). «Intérêt, conflit et pouvoir», in Images de l'organisation, Sainte-Foy, Presses de l'Université Laval, p. 213.

7 Emmanuel Joseph, dit l'abbé Sieyès. (1982) (1789). Qu'est-ce que le tiers État?, Paris, Presses universitaires françaises, p. 67.

8 De Koning, J. (2001). «Comment rendre les politiques actives du marché du travail plus efficaces ? Le rôle des organisations, de la mise en cuvre et de l'allocation optimale dans la politique active du marché du travail », in OCDE, Politiques du marché du travail et service public de l'emploi, Paris.

9 Proulx, M.-U. (2003). « Observer la nouvelle géographie », in Y. Leclerc, Y. Béland et al., La Voie citoyenne, pour renouveler le modèle québécois, Éditions Plurimédia, coll. Économie et Humanisme, p. 47.

10 OCDE (1998). Pour des politiques de l'emploi plus efficaces : la gestion locale, Paris, Organisation de coopération et de développement économiques.

11 OCDE (1999). Decentralising Employment Policy. New Trends and Challenges, Paris, The Venice Conference.

12 Favreau, L. (1999). Décentralisation du service public de l'emploi et initiatives locales: l'expérience québécoise, Université du Québec à Hull, Chaire en développement communautaire.

13 Rifkin, J. (1996). La fin du travail, Québec, Éditions du Boréal.

14 Boyer, R. et J.P. Durant (1994). L'après-fordisme, Paris, Syros.

15 Castel, R. (1994). Les métamorphoses de la question sociale, Paris, Fayard.

16 Angers, F.A. (1994). «Le plein emploi serait-il la solution au problème de la pauvreté ?» L'Action nationale, vol. LXXXIV, n 3, mars, p. 306.

17 Gorz, A. (1988). Métamorphose du travail, Paris, Galilée.

18 Grand'Maison, J. (2003). Questions interdites sur le Québec contemporain, Montréal, Fides, p. 75. 